documentary which aired on Channel 4 in 2018 - My Baby's Life: Who Decides? - which highlighted the ethical challenges in an increasingly technologically capable world. The programme raised issues in the region and demonstrated the need for healthcare professionals working with children with complex needs to draw together. In addition to developing skills in dealing with difficult decisions in paediatrics, the day has provided a bridge between academic work in this area and clinicians. As a result of the Difficult Decisions in Paediatrics forum, the region now has a paediatric Clinical Ethics Committee at which healthcare professionals can discuss cases at the University Hospital. Both the Difficult Decisions in Paediatrics Day and the regional Clinical Ethics Committee have received excellent feedback, and this work has enabled clinicians to move forward with increasingly complex decisions for the benefit of the children they work with.

\section{G360 MINORITY REPORT: CAN MINOR-PARENTS REFUSE MEDICAL TREATMENT FOR THEIR CHILD}

${ }^{1} \mathrm{HL}$ Turnham, ${ }^{2}$ A Binik, ${ }^{1} \mathrm{D}$ Wilkinson. ${ }^{1}$ Uherio Centre for Practical Ethics, Department of Philosophy, Oxford University, Oxford, UK; ${ }^{2}$ Department of Philosophy, McMaster University, Hamilton, Ontario, Canada

10.1136/archdischild-2020-rcpch.308

Infants are unable to make their own decisions or express their own wishes about medical procedures and treatments. They rely upon surrogates to make decisions for them. Who should be the decision-maker when an infant's biological parents are also minors?

$\mathrm{M}$, is a 15 year old girl who has a long-standing needle phobia, she gives birth to $\mathrm{B}$ and is assessed to be capable of caring for him. B is discharged into M's care on the second day of life. However, on day 7 the baby develops apnoeas and is brought to hospital by $M$ and her own mother, $G$, with whom they live. There are signs that B has sepsis. The paediatricians plan to insert an intravenous cannula (IV) and perform a lumbar puncture (LP) to exclude meningitis.

$\mathrm{M}$ consents to an IV for $\mathrm{B}$ and administration of antibiotics. However, she finds the process so distressing she refuses consent for $\mathrm{B}$ to undergo the LP.

The central questions raised by the case are whether minor-parents should make medical decisions on behalf of an infant, and if so, what are the limits to this decision-making authority? In particular, can they refuse treatment that might be considered best for the infant?

We examine different claims to parental decision-making authority; we argue that provided that minor-parents are capable of fulfilling their parental duties, they should have a right to make medical decisions for their infant.

We then examine the limits to minor parents' decision-making authority for their children. We argue that the restricted authority that teenagers are granted to make medical decisions for themselves looks very similar to the restricted autonomy of all parents. That is, they are permitted to make choices, but not harmful choices. Like all parents, minor parents must not abuse or neglect their children and must also promote their welfare. They have a right to make medical decisions for their infants within the same 'zone of parental discretion' that applies to adult parents. We conclude that adult and adolescent parents should have comparable decision-making authority for their infants.
G361

SERVICE EVALUATION OF MANAGEMENT OF CHILDREN AND YOUNG PEOPLE PRESENTING WITH ALCOHOL INTOXICATION TO A PAEDIATRIC EMERGENCY DEPARTMENT IN NORTHERN ENGLAND

M Dasarathi, M Livingstone. Paediatrics, South Tyneside and Sunderland NHS FT, Sunderland, UK

10.1136/archdischild-2020-rcpch.309

Aims To evaluate management of children and young people (CYP)aged 8-16 years presenting to a Paediatric emergency department(PED) in Northern England with alcohol intoxication and to identify any relevant service improvements.

Methods Retrospective analysis of electronic records of cases coded on Snowmed database as 'alcohol intoxication' or 'toxic effects of alcohol'. Data collected for overall number of PED admissions with alcohol intoxication over 5 years period from June 2013-May 2018. Data analysis for period June $2017-$ May 2018 included details on demographics, management, medical diagnosis/comorbidities, services referred into and readmission rate for CYP. Clinical management compared with 'Management of children and young people with an acute decrease in consciousness level'-clinical guideline by $\mathrm{RCPCH}$ (Royal College of Paediatrics and Child health).

Results 338 CYP aged 8-16 years attended PED with alcohol intoxication during above 5 years period. 25\% reduction noted in number of admissions in 2017-2018 as compared to 2013-2014. Detailed data analysis undertaken for 60 cases for the period June'17-May'18. Results showed female preponderance $(60: 40 \%)$. Observations and blood sugar levels documented in $100 \%$. Glasgow coma score (GCS) not documented in $15 \%$. Investigations included blood alcohol level (3\%), urine toxicology (8\%), Paracetamol blood level (13\%) and CT brain (23\%). 92\% had no underlying medical diagnosis. $74 \%$ discharged directly from PED, 26\% admitted to Paediatric ward. $80 \%$ referred to Youth Drug and Alcohol services (YDAP), 15\% to CYP services (mental health), 52\% had safeguarding liaison forms completed, $16 \%$ had Police involvement. 1/3rd of cases had red flags on electronic system (Looked after children, Child protection plan, MARAC). $16.6 \%$ re-attended, $60 \%$ of these had adverse social factors. Conclusions Overall management in PED appropriate, agreed to improve documentation on GCS. Consent required for YDAP referrals which may affect referral uptake. Direct liaison with YDAP is important to explore training for Paediatric staff to maximise support for this vulnerable group. Children are recognised to present at younger age (youngest age in our cohort was 11 years old). Use of electronic flagging systems can highlight vulnerable group at increased risk of readmission with alcohol intoxication. This can help explore further targeted interventions for this group.

\section{G362(P) SIMULATION TEACHING AS A MODALITY TO TEACH PALLIATIVE MEDICINE SKILLS IN A GENERAL PAEDIATRIC SETTING}

N Taylor, J Grimbley. Paediatric Department, James Cook University Hospital, Middlesbrough, UK

\subsection{6/archdischild-2020-rcpch.310}

Aims To assess the role of simulation teaching to teach paediatric palliative medicine (PPM) skills to the multidisciplinary team within a general paediatric setting. 
Methods Three PPM simulations were carried out in a general paediatric setting. Themes: acute presentation of a patient with no treatment ceiling decisions in place but parents not wishing for escalation of treatment; putting an advance care plan/DNACPR into place during acute presentation of a dying child and managing an expected death. Simulations were attended by medical and nursing staff with 2-3 staff actively participating and others observing. Two simulations were run with a high-fidelity mannequin and one with a low fidelity mannequin. Supporting items were used including DNACPR and EHCP (emergency health care plan) documentation, clinic letters/ward round entries and drug prescriptions.

All participants were asked to complete feedback at the end of each session and active participants were contacted following the session for further feedback. A five point Likert scale was used to rate enjoyment, organisation, quality and relevance of session and likelihood of attending further simulation teaching in PPM. A 10-point confidence scale was used to rate confidence in dealing with PPM scenarios and confidence in talking to families about death both before and after the scenario. Additional comments were also collected.

Ethical approval was not required.

Results Participants reported they enjoyed the sessions (mean score $4.7 / 5$; range $0-5$ ); felt they were well organised $(4.7 / 5 ; 1-5)$ and of high quality $(4.7 / 5 ; 1-5)$. They felt sessions were relevant to their training $(4.5 / 5 ; 1-5)$ and that they would attend further simulation training in the area of PPM (mean 4.8/5; range 1-5). Confidence in managing PPM scenarios increased by a mean of 2.4 confidence points (range $0-5$ ) and confidence in managing conversations around death increased 1.8 confidence points (range 0-5).

Conclusion Simulation training to teach PPM skills to the multi-disciplinary paediatric team was acceptable to participants and improved participants' confidence in managing PPM scenarios and conversations around death.

\section{G363(P) SHARE OUR LEARNING EXPERIENCE AND APPROACH USED TO DEAL WITH A CHALLENGING COMPLEX CASE INCLUDING SAFEGUARDING CONCERNS AND ETHICAL ISSUES IN A COMMUNITY SETTING}

${ }^{1} \mathrm{R}$ Jainer, ${ }^{1,2}$ E Isles. 'Department of Paediatrics, University Hospitals of Birmingham NHS Trust, Solihull, UK; ${ }^{2}$ Community Nursing Team, University Hospitals of Birmingham NHS Trust, Solihull, UK

\subsection{6/archdischild-2020-rcpch.311}

Aims Share our learning experience of dealing with a challenging complex case with life limiting condition called Canavans disease in a Community setting.

It was an acute life threatening safeguarding concern on background of long standing concerns by health professional on a 17 year old. He had complex health care needs including respiratory support and gastrostomy feeds. He is dependent on adults for all his care and administration of medications. He was on child in need plan due to long standing concerns.

Family suddenly expressed a wish to take him on a long haul flight to attend a family wedding to meet their own emotional and social needs.
Method Information gathering from varied professionals. Multiagency meetings and sharing information transparently.

Presentation at Regional palliative care meeting, no consensus reached. Risks assessment completed and discussed with family about safety, dignity, pain relief and risk of deterioration on flight and empathised that despite efforts to mitigate concerns would not be in his best interest.

Family was not in agreement with decision of professionals

Escalated concerns with safeguarding lead and advice from legal team taken.

Best interest team meeting was arranged.

Result Decision in best interest of the young person not to fly as risks outweighed the benefits.

Young person was abandoned by family without appropriate carer to attend to his needs. He was then made ward of court and admitted in hospital till residential placement was arranged and transited to adult services.

Conclusion Despite adherence to principles of palliative care which was holistic, patient centred care and transition to adult services in a community setting, we are faced with ethical and legal challenges.

However, challenge was dealt with clinical leadership and team, effective communication skills and peer support and all having a common goal of compassion and ensuring safety of the young person.

This also prevented an impending safeguarding practice review

Recommendations Sharing of more such cases in a wider forum to develop better understanding for clinicians about what avenues are accessible. Having training days jointly organised by Paediatric palliative care with input from safeguarding leads would help clinicians acquire the needed skills for dealing with such complex ethical cases.

\section{G364(P) WHAT SIGNS OR SYMPTOMS INDICATE A CHILD IS LIKELY TO DIE WITHIN MONTHS OR DAYS AND ARE THEY BEING USED WITHIN THIS HOSPITAL?}

${ }^{1} S$ Lovegrove, ${ }^{2,3} \mathrm{~J}$ Manning, ${ }^{2} \mathrm{~T}$ Wolff. ${ }^{1}$ School of Medicine, University of Nottingham, Nottingham, UK; ${ }^{2}$ Nottingham Children's Hospital, Nottingham University Hospitals, Nottingham, UK; ${ }^{3}$ School of Health Sciences, University of Nottingham, Nottingham, UK

10.1136/archdischild-2020-rcpch.312

Background Clinicians must be able to spot when children with life-limiting and life- threatening conditions might be approaching the last 6-12 months of life, to allow time for advance care planning to be put in place. As yet, no prognostic indicator tool exists for paediatric palliative care. The Chameleon children's end of life (EoL) care quality improvement project promoted the use of an EoL care prompt during 2018. The purpose of this audit was to then determine if the addition of prognostic indicators to the prompt tool may improve its use and thus the timely implementation of essential interventions.

Objectives To determine locally used prognostic indicators for the last 6-12 months of a child's life within this Children's Hospital. To retrospectively ascertain if they have been used and if appropriate interventions have been completed from the EoL care prompt.

Methods 12 healthcare professionals were interviewed to find locally used prognostic indicators. Children who died an expected death as a result of either an oncological or 